\title{
Co-exploring Actuator Antagonism and Bio-inspired Control in a Printable Robot Arm
}

\section{Stoelen, MF}

http://hdl.handle.net/10026.1/6711

\subsection{7/978-3-319-43488-9_22 \\ Lecture Notes in Computer Science \\ Springer}

All content in PEARL is protected by copyright law. Author manuscripts are made available in accordance with publisher policies. Please cite only the published version using the details provided on the item record or document. In the absence of an open licence (e.g. Creative Commons), permissions for further reuse of content should be sought from the publisher or author. 


\title{
Co-Exploring Actuator Antagonism and Bio-Inspired Control in a Printable Robot Arm
}

\author{
Martin F. Stoelen ${ }^{1}$, Fabio Bonsignorio ${ }^{2}$, and Angelo Cangelosi ${ }^{1}$ \\ 1 Centre for Robotics and Neural Systems, Plymouth University, Plymouth, UK \\ \{martin.stoelen, a.cangelosi\}@plymouth.ac.uk \\ 2 The BioRobotics Institute, Scuola Superiore Sant'Anna, Pisa and Heron Robots, \\ Genova, Italy fabio.bonsignorio@sssup.it
}

\begin{abstract}
The human arm is capable of performing fast targeted movements with high precision, say in pointing with a mouse cursor, but is inherently 'soft' due to the muscles, tendons and other tissues of which it is composed. Robot arms are also becoming softer, to enable robustness when operating in real-world environments, and to make them safer to use around people. But softness comes at a price, typically an increase in the complexity of the control required for a given task speed/accuracy requirement. Here we explore how fast and precise joint movements can be simply and effectively performed in a soft robot arm, by taking inspiration from the human arm. First, viscoelastic actuator-tendon systems in an agonist-antagonist setup provide joints with inherent damping, and stiffness that can be varied in real-time through co-contraction. Second, a light-weight and learnable inverse model for each joint enables a fast ballistic phase that drives the arm close to a desired equilibrium point and co-contraction tuple, while the final adjustment is done by a feedback controller. The approach is embodied in the GummiArm, a robot which can almost entirely be printed on hobby-grade 3D printers. This enables rapid and iterative co-exploration of 'brain' and 'body', and provides a great platform for developing adaptive and bio-inspired behaviours.
\end{abstract}

Keywords: Bio-inspiration, learnable models, agonist-antagonist joints, variable stiffness, 3D printing, targeted movements

\section{Introduction}

This paper concerns a bio-inspired robot arm, the GummiArm. See Fig. 1. The robot is based on a set of principles drawn from the human and animal sensorimotor system. These principles include:

1. Agonist-antagonist actuators with control of joint equilibrium point and cocontraction. The equilibrium point hypothesis has been used to predict multijoint human trajectories [1], and has been shown to lead to fast point-to-point movements in biomechanical simulations [2]. 

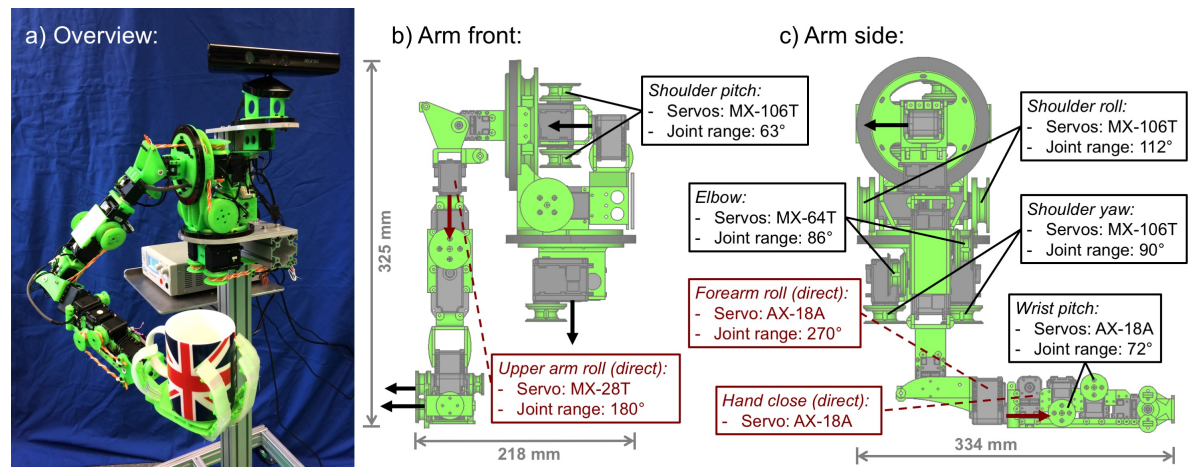

Fig. 1. The GummiArm v2.1.0. All light green parts are printable on hobby-grade 3D printers, while the joints are actuated by Dynamixel (Robotis Inc, Irvine, CA, USA) digital servos. The 5 agonist-antagonist joints provide inherent damping, impact robustness, and stiffness adjustment in real-time, through the composite viscoelastic tendons seen in orange and white. 3 further joints are directly driven by servos, the upper arm roll, forearm roll, and hand close. a): The arm mounted on an aluminium frame, with a Kinect sensor (Microsoft, Redmond, WA, USA) on a pan mechanism. b) and c): Annotated front and side views, respectively. Thick filled-in arrows indicate the joint $\hat{z}$ axes.

2. Viscoelastic actuator-tendon system. Humans exploit co-contraction of the viscoelastic muscle-tendon system both during movements and after movement completion for achieving accuracy [3]. Here we show that we can adjust stiffness through co-contraction of rubber tendons with non-linear stiffness, and use movement-dependent excitation of co-contraction to further control unwanted end-point oscillations. The viscoelasticity provides damping.

3. Learnable inverse joint models for feedforward control of rapid point-to-point movements in joint-angle space. There is extensive work on inverse models for movement control in the brain [4]. We here use learnable inverse models for the joints to generate a ballistic phase of movement towards a given joint angle and co-contraction level. A second phase uses a feedback controller to compensate for any model deviations.

4. A concurrent approach to the design of 'brain' and 'body', enabled by a printable platform with open-source hardware and software. The robot structure is printable on hobby-grade $3 \mathrm{D}$ printers, and the overall platform cost is reasonably low (less than $\$ 5000$ ) for a $7+1$ Degree Of Freedom (DOF) arm with variable stiffness. The passive compliance makes it robust to impacts, and a broken part can be 3D printed (and potentially improved upon) quickly.

\section{Related Work}

Soft materials can afford new capabilities in safety, speed and agility of robotic agents [5]. Soft materials also have the potential to reduce the algorithmic com- 
plexity if the 'body' and 'brain' are developed together [6]. On one end of the 'soft' spectrum we find artificial octopus arms [7], providing extreme dexterity and compliance. On the other end are robots with stiff links, but elastic elements connecting the links and actuators. That is, series elastic actuators [8]. As an example, a low-cost compliant actuator was developed by Quigley, Asbeck and Ng [9], with a series elastic setup for the main actuators. The Polyurethane elastic elements provided compliance and some damping, but could not be varied in real-time. Another example is the Baxter light industrial robot (Rethink Robotics, Boston, USA). The inability to increase physical stiffness (and damping) can make such robots hard to control on fast point-to-point movements.

However, Variable Stiffness Actuators (VSA) are gradually becoming commonplace [10]. Benefits over traditional stiff robot actuators include safety, for humans, robots and the environment, but also performance [11]. The VSACubebot [12] is a great example of a low-cost and flexible VSA. The standard modular design can simplify the design of a VSA arm, but it also means the actuators will typically have to be placed at the joints. The DLR hand arm system is a full size VSA arm with extensive use of tendons [13]. It is aimed at human levels of scale and performance, and therefore also has a high complexity. A key issue with introducing elastic elements into the actuation loop is that end-point oscillations can be hard to dampen. Advanced torque control strategies is one way to approach this problem [14], but typically requires an accurate robot model. Variable damping can also be achieved through physical means in the actuator. For example an electrically damped actuator [15]. The CompAct anthropomorphic actuator is also able to vary the physical damping, through piezo-electric clutches and an advanced sliding-mode control [16].

If exact models of the body cannot easily be pre-defined by the designer, such models can perhaps be learnt as part of the 'development' of the robot [17]. Among the platforms exploring this general direction is Roboy [18], a tendondriven humanoid robot with passive compliance and force sensing directly in the muscle units. The iCub [19], one of the most popular platforms for developmental robotics, is also tendon-driven. However, it lacks passive compliance, and is aimed at a much higher cost and complexity level. A model-free approach for damping VSAs with a step change at just the right point in the oscillations shows promise [20], and should be possible to combine with the work presented here in the future.

\section{The GummiArm}

\subsection{An Easily Evolvable Arm}

The GummiArm is a 7+1 DOF robot arm, and is an open-source project available at: http://mstoelen.github.io/GummiArm/. See Fig. 1. The structure of the GummiArm consists of plastic parts connected to Dynamixel digital servos of Robotis Inc (Irvine, CA, USA). This design feature was inspired by the Robotis Bioloid robots and the Poppy Project [21], but with the addition of variable stiffness. The proportions of the arm (except the current hand) are equivalent to 
a 50th percentile female human [22]. The servos are joined by PLA-based plastic parts that can be printed on hobby-grade 3D printers. PLA is safe and cheap, and the parts can be made surprisingly light and strong due to the matrix-like internal structure. The total mass of the $7+1$ DOF arm below the shoulder is 1.1 $\mathrm{kg}$ (excluding the hand), and the total mass of the arm is around $3 \mathrm{~kg}$. Less than $1 \mathrm{~kg}$ of PLA plastic is needed to print the current version of the arm (v2.1.0).

The combination of fully open source software and hardware (with the exception of the servos) makes it possible to do a concurrent design of the soft arm itself with the control and adaptation algorithms. For example by quickly modifying the 3D printed parts of a joint to handle bigger tendons, while making corresponding changes to accommodate the higher stiffness in the arm control. Such changes can be made on the order of minutes and hours, leading to fast iterative improvements that explore the full design space of hardware and software. A bit like evolution on a small scale, with the designer in the loop.

\section{$3.2 \quad$ Agonist-Antagonist Joints}

Agonist-antagonist joints have been explored extensively for bio-inspired robotic arms. For example with two opposing pneumatic actuators, such as the McKibben type [23]. Such actuators require an external compressor however, and can be hard to control. The GummiArm has 5 agonist-antagonist joints with electric actuation, see Fig. 1. Each agonist-antagonist joint has two Dynamixel servo actuators operating the uni-directional tendons via pulleys, and one encoder on the joint axis. The tendons are based on a Filaflex $2.85 \mathrm{~mm}$ filament from Recreus (La Torreta, Spain), and their elasticity provide the arm passive compliance.

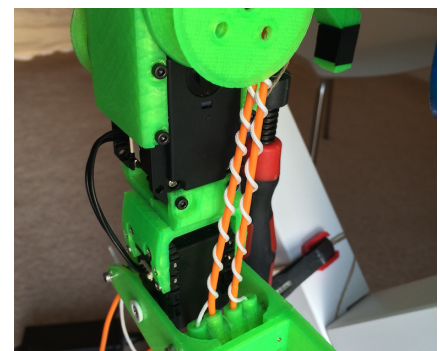

(a) 2 composite tendons on the biceps servo pulley.

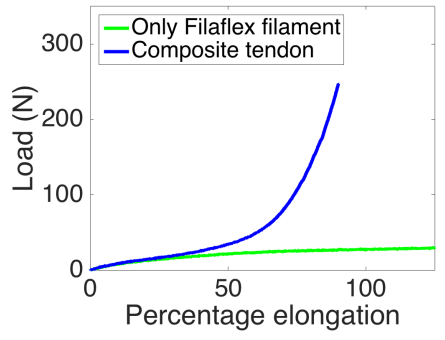

(b) Load (y-axis) vs elongation ( $\mathrm{x}$ axis) of tendons.

Fig. 2. The composite tendon design, based on a soft $2.85 \mathrm{~mm}$ Filaflex filament (Recreus, La Torreta, Spain) and a stiff $1.5 \mathrm{~mm}$ nylon thread twinned around it.

A quadratic force-length relationship is desirable in tendons used for agonistantagonist joints. This allows independent control of stiffness and equilibrium without sensory feedback, as shown in [24]. To approach such behaviour we 
emulated typical rubber compensators for mooring lines on boats. That is, a much less flexible nylon line was twinned around the Filaflex filament, as seen in Fig. 2(a). As can be seen in Fig. 2(b) this composite tendon design has an increase in stiffness with elongation, as the nylon line gradually straightens out. The tensile testing was performed on a Instron (Wycombe, United Kingdom) 5582 frame with a static $100 \mathrm{kN}$ load cell (Instron UK195) on a $190 \mathrm{~mm}$ specimen. For the tensile testing a pitch of 0.1 per mm was used.

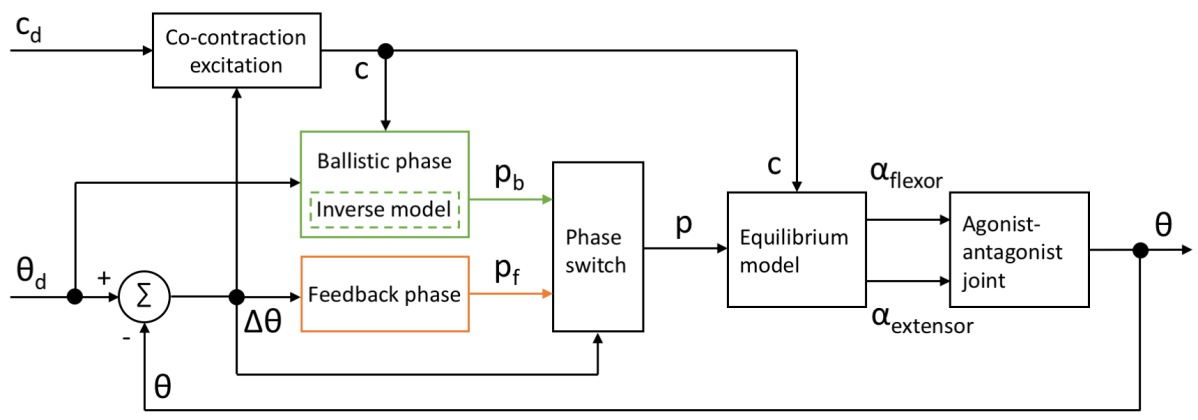

Fig. 3. The control architecture for the agonist-antagonist joints. The joint controller is provided with a desired joint angle $\theta_{d}$ and co-contraction level $c_{d}$ (from 0 to $100 \%$ ). For large desired changes in joint angle (here $|\Delta \theta|>15^{\circ}$ ) a switching mechanism activates the ballistic phase. The co-contraction $c$ is also excited proportionally to the desired change in joint angle $\Delta \theta$, to provide stabilization towards the end of the movement. The ballistic phase aims for a high percentage of the desired joint angle change (here $85 \%$ ), and aims to get there with the excited co-contraction level. A desired equilibrium point $p_{b}$ is generated with an inverse joint model and fed forward. An equilibrium model relates the equilibrium point and co-contraction to the required actuation commands, which are here the angles of the actuator pulleys $\left(\alpha_{\text {flexor }}\right.$ and $\left.\alpha_{\text {extensor }}\right)$. When a threshold percentage of $\Delta \theta$ is passed (here 50\%) the feedback controller takes over, correcting for discrepancies between the model and the real situation of the joint.

\subsection{Combined Ballistic/Feedback Control}

A dual-phase control architecture is used for controlling the GummiArm on fast point-to-point joint movements. See Fig. 3. Referring to the 'Equilibrium model' box, the two servo actuator angles $\alpha_{\text {flexor }}$ and $\alpha_{\text {extensor }}$ for a joint are assumed to scale linearly with equilibrium point $p$ and co-contraction $c$. See Eq. 1.

$$
\begin{aligned}
& \alpha_{\text {flexor }}=p \frac{\gamma}{4}-c \frac{\pi}{2}, \\
& \alpha_{\text {extensor }}=p \frac{\gamma}{4}+c \frac{\pi}{2} .
\end{aligned}
$$

Note that the equilibrium point here is a virtual joint feature, and any deviations from this assumption is attempted corrected through the joint calibration. 
For simplicity we here assume step-changes to equilibrium point in joint-space, rather than the task-space trajectories with explicit velocity profiles used by among other Flash [1]. The equilibrium point $p$ ranges from -1 to 1 , and is assumed to influence half the actuator range $\gamma$. This range was $270^{\circ}$ for the wrist joint (AX-18 servos), $360^{\circ}$ for the elbow (MX-64T servos) and shoulder pitch (MX-106T servos), and $720^{\circ}$ for the remaining joints (MX-106T servos). The co-contraction $c$ ranges from 0 to 1 ( $0 \%$ to $100 \%$ ), corresponding to $\pm 90^{\circ}$ of range on the actuator servos.

The co-contraction was set according to the desired $c_{d}$, but was also excited by large commanded changes in joint angle from the actual, when a distinct point-to-point command was received. Refer to the 'Co-contraction excitation' box in Fig. 3. As can be seen in Eq. 2 the ballistic component $c_{b}$ was scaled proportionally with the absolute value of $\Delta \theta$.

$$
\begin{aligned}
& c_{b}=k|\Delta \theta|, \\
& c_{e}=c_{b}-c_{d} .
\end{aligned}
$$

The co-contraction $c$ was set according to: $c=c_{d}+c_{e}$. Here $c_{e}$ was reduced every iteration by the factor $\mu$, with $0<\mu<1$, according to: $c_{e}=c_{e} \mu$. Thus the co-contraction is gradually reduced to the desired value $c_{d}$, relaxing the joint after the movement. The constants $k$ and $\mu$ were adjusted for this to occur, with $k=0.035$ and $\mu=0.0015$ for a $60 \mathrm{~Hz}$ update rate of the control system.

As described in Fig. 3, the ballistic phase was initiated if $|\Delta \theta|>15^{\circ}$. Ballistic movements, with little or no online sensory feedback, have been widely studied in humans. They typically exhibit a characteristic 'triphasic' burst of activity in agonist and antagonist muscles, for example in fast thumb flexion [25]. In our current work the inverse model, described in Section 3.4, was used to obtain the equilibrium point for the ballistic movement, given the co-contraction $c$. The feedback phase (see corresponding box in Fig. 3) was set to take over when more than $50 \%$ of the joint angle movement had been completed. See 'Phase switch' box in Fig. 3. A PID feedback controller was used, tuned to provide reasonable performance over the full range of co-contraction levels available. To help reduce overshoot, the ballistic phase was aimed at $85 \%$ of the actual $\Delta \theta$ required.

\subsection{Inverse Joint Model for Ballistic Phase}

The term 'inverse model' is used to denote transformations from desired object movements to motor commands [4]. The ballistic phase described above requires a mapping from a desired joint angle and co-contraction to the corresponding equilibrium point $p_{b}$. That is, what muscle lengths (here the servo actuator angles $\alpha_{\text {flexor }}$ and $\alpha_{\text {extensor }}$ ) are required to reach a certain joint pose with a given amount of stiffness. This mapping would in general depend on the forces acting on the joint, and thus also the pose of the full arm, any payload held in the hand, any interaction with the environment, and the dynamics of the movement. We here assume a much simpler model, which provides a mapping 
under quasi-static conditions around the resting pose of the arm seen in Fig. 1. We show that such a simple model is sufficient in many cases, when combined with a feedback phase, the intrinsic damping of the viscoelastic tendons used, and the movement-dependent excitation of co-contraction.

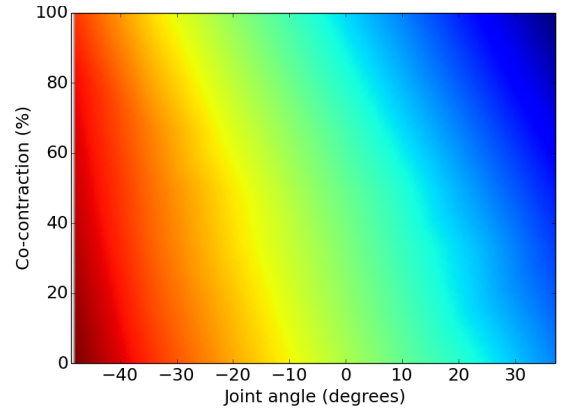

(a) Elbow joint.

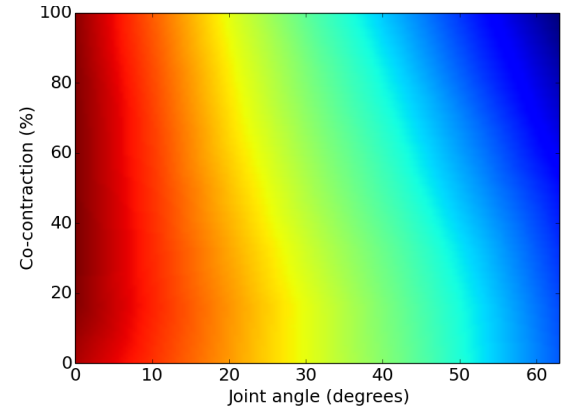

(b) Shoulder pitch joint.

Fig. 4. The mappings for the inverse models of the elbow and shoulder pitch joints. Colour represents the output of the model, the equilibrium point $p$.

The inverse model for the elbow and shoulder pitch joints are visualized in Fig. 4. The inverse models were aquired using a calibration procedure for each joint. The joint was moved through the full joint range, stopping at 7 quasi-static poses (joint angles), each at 7 different levels of co-contraction (from 0 to 100\%). A linear interpolation could then be used to obtain values for $p_{b}$ spanning the convex hull of the 49 calibration points. That is, the model assumes linearity between the 49 points obtained, although a finer sampling scheme could be used if required. The Python scipy.interpolate.griddata function was used. The full calibration procedure takes less than 5 minutes for each joint. An estimate for the appropriate $p_{b}$ value could be obtained in less than $1 \mathrm{~ms}$ for each joint, on an Intel i7 5960X running at $3 \mathrm{MHz}$. An interesting feature of these inverse models is that they can be adjusted in real-time, during quasi-static poses. Such learning could help adjust the arm performance to the task context, for example if always holding an object of a certain mass on a given task.

\section{Experimental Results}

\subsection{Exploring Co-Contraction}

The ability to co-contract opposing actuators is the key feature of agonistantagonist joints. A quasi-static loading setup was created for the elbow joint. The upper arm was locked in place, while the lower arm was replaced with a rigid beam with multiple attachment points for weights, from $70 \mathrm{~mm}$ to $200 \mathrm{~mm}$ from the joint axis, and at $10 \mathrm{~mm}$ intervals. The actuator was commanded to a passive 
horizontal pose. Three different weights $(0.1 \mathrm{~kg}, 0.5 \mathrm{~kg}$, and $1.5 \mathrm{~kg})$ were then attached at different distances from the joint axis to generate a set of torques up to almost $3 \mathrm{Nm}$. The passive deflection of the joint was then recorded with the AX-12A encoder. This process was repeated three times for the 3 weights and the 14 distances. The same procedure was repeated for 5 different stiffness levels, from $0 \%$ to $100 \%$. See Fig. 5 for the results. The maximum torque feasible for the elbow joint with the MX-64T servo was close to $3 \mathrm{Nm}$, reducing somewhat with the highest stiffness setting. It can be seen that the amount of deflection for a given external torque can be changed considerably by the co-contraction. The deflections possible are also quite high for a VSA [26], exceeding $45^{\circ}$ at high external torques with the $0 \%$ stiffness setting. Such 'softness' is an interesting feature when having robots explore autonomously the physical world in developmental experiment paradigms. A 100\% change in stiffness can for most joints be done in less than 0.5 seconds.

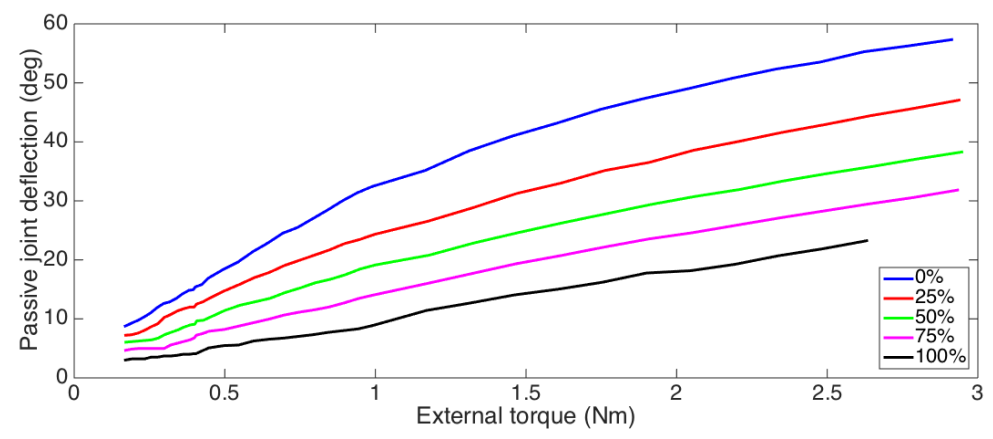

Fig. 5. Passive joint deflection (y-axis) of the elbow joint from applying an external torque (x-axis) under quasi-static conditions, for different values of commanded cocontraction. Deflection corresponding to elbow extension.

\subsection{Fast Joint-Space Movements}

The step response of the shoulder pitch joint was explored, with feedback control only, and with the bio-inspired two-phase ballistic/feedback control. The joint was mounted as part of the full arm, and all other joints were kept passively at the resting pose (see Fig. 1). The shoulder pitch joint was moved through $3 / 5$ of full joint range, from close to body to shoulder abduction, and back again. As can be seen in Fig. 6, both controllers showed good tracking of the desired joint angle with $100 \%$ co-contraction, and little overshoot and oscillations. The ballistic/feedback controller did show a superior response time, but the difference was small and requires further investigation. However the ballistic/feedback controller was able to provide very good tracking also for down to $0 \%$ co-contraction at start, while the feedback controller showed increasing levels of oscillations. For 
both cases a PID-type feedback controller was used, but a simpler PD controller would likely suffice, as there is little steady-state error.

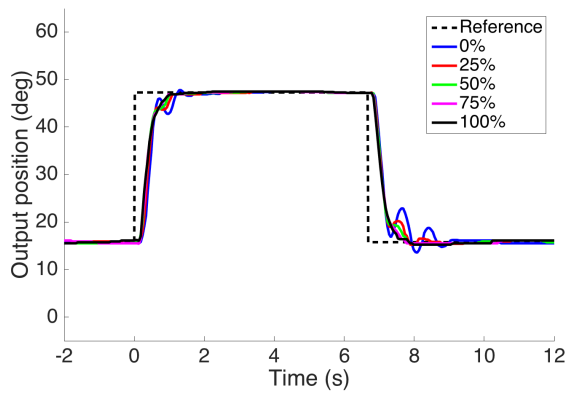

(a) Feedback control only.

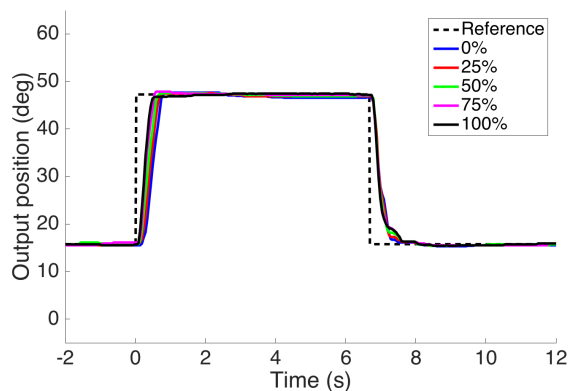

(b) Ballistic/feedback control.

Fig. 6. Step responses of the shoulder pitch joint with elbow at resting pose in Fig. 1(b), for different levels of co-contraction. Zero degrees corresponds to resting pose for shoulder pitch joint. Average of 3 attempts for each trajectory shown.

The full arm was also assessed on point-to-point movements, to compare the controllers when there are un-modelled interactions between the moving joints. All joints were commanded to move as fast as possible to the joint angles corresponding to a finish pose. Note that no inter-joint coordination was performed, each joint moved as quickly as possible. Such a movement can cause large interacting forces between joints, making it harder for the joint-level controllers. As can be seen in Fig. 7, the feedback controller suffered from these interactions during the movement, and had a considerable amount of oscillations towards the end of the movement. The ballistic/feedback controller performed better, also with $0 \%$ co-contraction at start (not shown). The achievable speed of movement was also higher for the ballistic/feedback controller. This can be evaluated visually by the fewer 'shadows' seen in the intermediate stages of movement in the long-exposure image in Fig. 7(b). Further tests are required to generalise about these results, and to better understand the factors influencing performance.

\subsection{Teleoperation with Physical Interaction}

The GummiArm is utilising the Robot Operating System (ROS) for all its functionality. A differential inverse kinematics solver based on the OROCOS [27] KDL library is also implemented, allowing teleoperation of the arm. While teleoperating the co-contraction can be adjusted freely by the operator, making the arm joints loose or stiff. The arm can also be commanded in a passive mode, where the equilibrium position of each joint is moved without controlling for the exact joint angle. This is very useful when interacting with physical objects, as the arm complies naturally to the forces experienced. Fig. 8 shows screenshots 


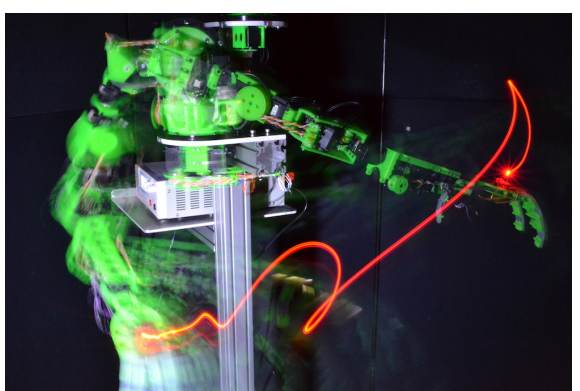

(a) Feedback control only.

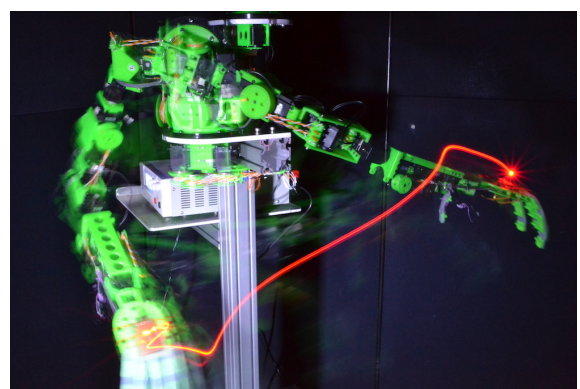

(b) Ballistic/feedback control.

Fig. 7. Fast point-to-point movement in joint space with the whole arm, with $100 \%$ co-contraction. Note that the movements are generated in joint-space, not as a straightline Cartesian trajectory. The goal is to achieve the movement as fast as possible, settling within a minimum tolerance of the desired finish pose. Images created with a long-exposure of 6 seconds, and strobe lighting at $8.5 \mathrm{~Hz}$. Red LED mounted on hand.

of a continuous sequence where the robot shows its ability to absorb impacts, to be teleoperated accurately with high stiffness, to write on a keyboard, and to open a drawer while moving in the passive mode. The full video can be accessed here: https://youtu.be/_syFAQBrgio.

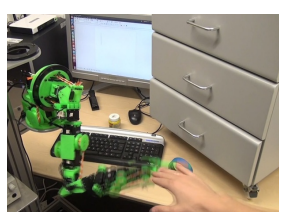

(a) Absorbing physical impacts.

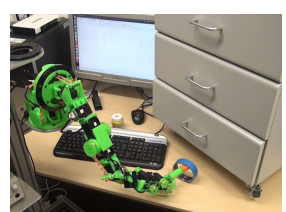

(b) Performing precision move.

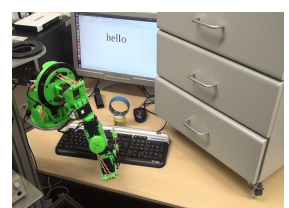

a (c) Typing on a com- ( puter keyboard.

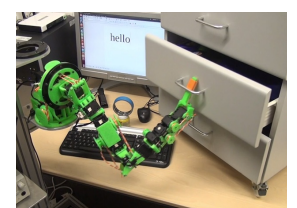

(d) Opening an office drawer.

Fig. 8. Example teleoperation task with physical interaction.

\section{Conclusions}

Co-exploring 'body' and 'brain' seems fruitful when investigating adaptive behaviours. We believe the GummiArm platform is highly suitable for this purpose, and can be of use to researchers at the intersection of biology, cognitive science and human motor control with robotics. The arm has a high robustness and a low lifetime cost, given the low-cost printable PLA structure, the passive compliance of the tendons, and since a non-specialist user can quickly fix and improve any pieces that do break. A key element is the agonist-antagonist joints driven 
by accurate digital servos, and with viscoelastic composite tendons. The inherent damping and the ability to adjust stiffness in real-time helps simplify joint control. The dual phase ballistic/feedback controller was inspired by the way humans can quickly move the hand close to a target with a ballistic movement, then refine the pose with sensory feedback. It has a low algorithmic complexity, in essence relying on distributed and learnable inverse models in the joints, and simple switches, but enables the GummiArm to perform fast and accurate joint movements. Together with the ability to interact safely with the physical environment, this makes for interesting possibilities in robot self-exploration. We hope to exploit this, and to integrate more adaptive and context-sensitive behaviours in the arm, in the near future. We would also like to explore scalable equilibrium point trajectories in task space [1].

Acknowledgments. This work was funded by a Marie Curie Intra-European Fellowship within the 7th European Community Framework Programme (DeCoRo FP7-PEOPLE-2013-IEF).

\section{References}

1. Flash, T., "The control of hand equilibrium trajectories in multi-joint arm movements", Biological cybernetics, vol. 57, no. 4-5, pp. 257-274, 1987.

2. Kistemaker, D.A., Van Soest, A.K.J. and Bobbert, M.F., "Is equilibrium point control feasible for fast goal-directed single-joint movements?", Journal of Neurophysiology, vol. 95, no. 5, pp. 2898-2912, 2006.

3. Gribble, P.L., Mullin, L.I., Cothros, N. and Mattar, A., "Role of cocontraction in arm movement accuracy", Journal of neurophysiology, vol. 89, no. 5, pp. 2396-2405, 2003.

4. Wolpert, D.M., Miall, R.C. and Kawato, M., "Internal models in the cerebellum", Trends in cognitive sciences, vol. 2, no. 9, pp. 338-347, 1998.

5. Rus, D., and Tolley, M.T., "Design, fabrication and control of soft robots", Nature, vol. 521, no. 7553, pp. 467-475, 2015.

6. Pfeifer, R., Lungarella, M., and Iida, F., "The challenges ahead for bio-inspired 'soft' robotics", Communications of the ACM, vol. 55, nu. 11, pp. 76-87, 2012.

7. Mazzolai, B., Margheri, L., Cianchetti, M., Dario, P., and Laschi, C., "Soft-robotic arm inspired by the octopus: II. From artificial requirements to innovative technological solutions," Bioinspiration \& biomimetics, vol. 7, no. 2, 025005, 2012.

8. Pratt, G.A. and Williamson, M.M., "Series elastic actuators", IEEE/RSJ International Conference on Intelligent Robots and Systems (IROS), vol. 1, pp. 399-406, 1995.

9. Quigley, M., Asbeck, A. and Ng, A., 2011, May. "A low-cost compliant 7-DOF robotic manipulator", IEEE International Conference on Robotics and Automation (ICRA), pp. 6051-6058, 2011.

10. Vanderborght, B., et al., "Variable impedance actuators: A review", Robotics and Autonomous Systems, vol. 61, no.12, pp. 1601-1614, 2013.

11. Bicchi, A., Tonietti, G., Bavaro, M., and Piccigallo, M., "Variable stiffness actuators for fast and safe motion control", in The Eleventh International Symposium on Robotics Research, pp. 527-536, Springer Berlin Heidelberg, 2005. 
12. Catalano, M.G., Grioli, G., Garabini, M., Bonomo, F., Mancini, M., Tsagarakis, N., and Bicchi, A., "VSA-CubeBot: A modular variable stiffness platform for multiple degrees of freedom robots", IEEE International Conference on Robotics and Automation (ICRA), pp. 5090-5095, Shanghai, China, 2011.

13. Grebenstein, M., et al., "The DLR hand arm system", IEEE International Conference on Robotics and Automation (ICRA), pp. 3175-3182, 2011.

14. Petit, F., Dietrich, A. and Albu-Schaffer, A., "Generalizing Torque Control Concepts: Using Well-Established Torque Control Methods on Variable Stiffness Robots", IEEE Robotics \& Automation Magazine, vol. 22, no. 4, pp. 37-51, 2015.

15. Radulescu, A., Howard, M., Braun, D.J. and Vijayakumar, S., "Exploiting variable physical damping in rapid movement tasks", IEEE/ASME International Conference on Advanced Intelligent Mechatronics (AIM), pp. 141-148, 2012.

16. Kashiri, N., Tsagarakis, N.G., Van Damme, M., Vanderborght, B. and Caldwell, D.G., "Proxy-Based Sliding Mode Control of Compliant Joint Manipulators", Informatics in Control, Automation and Robotics, pp. 241-257, Springer International Publishing, 2016.

17. Cangelosi, A., and Schlesinger, M., "Developmental robotics: From babies to robots", MIT Press, 2015.

18. Pfeifer, R., Marques, H.G. and Iida, F., "Soft robotics: the next generation of intelligent machines", in Proceedings of the Twenty-Third international joint conference on Artificial Intelligence, pp. 5-11, 2013.

19. Metta, G., Sandini, G., Vernon, D., Natale, L., and Nori, F., "The iCub humanoid robot: an open platform for research in embodied cognition". In Proceedings of the 8th workshop on performance metrics for intelligent systems, pp. 50-56, 2008.

20. Petit, F., Ott, C. and Albu-Schaffer, A., "A model-free approach to vibration suppression for intrinsically elastic robots", IEEE International Conference on Robotics and Automation (ICRA), pp. 2176-2182, 2014.

21. Lapeyre, M., Rouanet, P., Grizou, J., Nguyen, S., Depraetre, F. et al., "Poppy Project: Open-Source Fabrication of 3D Printed Humanoid Robot for Science, Education and Art", Digital Intelligence 2014, Sep 2014, Nantes, France. pp. 6, 2014. $<$ hal-01096338>

22. NASA, "Man-Systems Integration Standards - Revison B". National Aeronautics and Space Administration: Houston, USA. 1995. Available at http://msis.jsc.nasa.gov/

23. Chou, C.P. and Hannaford, B., "Measurement and modeling of McKibben pneumatic artificial muscles", IEEE Transactions on Robotics and Automation, vol. 12, no. 1, pp. 90-102, 1996.

24. Ham, R.V., Sugar, T.G., Vanderborght, B., Hollander, K.W., and Lefeber, D., "Compliant actuator designs", IEEE Robotics \& Automation Magazine, vol. 16, no. 3, pp. 81-94, 2009.

25. Hallett, M.A.R.K. and Marsden, C.D., "Ballistic flexion movements of the human thumb", The Journal of Physiology, vol. 294, pp. 33-50, 1979.

26. Grioli, G., et al., "Variable stiffness actuators: The user's point of view", The International Journal of Robotics Research, vol. 34, no. 6, pp. 727-743, 2015.

27. Bruyninckx, H., "Open robot control software: the OROCOS project", IEEE International Conference on Robotics and Automation (ICRA), pp. 2523-2528, 2001. 